$05,10,11$

\title{
Фазовая диаграмма модели Поттса с числом состояний спина $q=4$ на гексагональной решетке
}

\author{
() А.К. Муртазаев ${ }^{1,2}$, М.К. Мазагаева ${ }^{1}$, М.К. Рамазанов ${ }^{1,2}$, М.А. Магомедов ${ }^{1,2}$, А.А. Муртазаева ${ }^{1}$ \\ ${ }^{1}$ Институт фризики Дагестанского федерального исследовательского центра РАН, \\ Махачкала, Россия \\ ${ }^{2}$ Дагестанский федеральный исследовательский центр РАН, \\ Махачкала, Россия \\ E-mail: sheikh77@mail.ru
}

Поступила в Редакцию 24 декабря 2020 г.

В окончательной редакции 24 декабря 2020 г.

Принята к публикации 19 января 2021 г.

На основе алгоритма Ванга-Ландау методом Монте-Карло выполнены исследования магнитных структур основного состояния, фазовых переходов и термодинамических свойств двумерной ферромагнитной модели Поттса с числом состояний спина $q=4$ на гексагональной решетке с конкурирующими обменными взаимодействиями. Исследования проведены для величины взаимодействия вторых ближайших соседей в диапазоне $0.0 \leq r \leq 1.0$. Показано, что учет антиферромагнитных взаимодействий вторых ближайших соседей приводит к нарушению магнитного упорядочения. Построена фазовая диаграмма зависимости критической температуры от величины взаимодействия вторых ближайших соседей. Проведен анализ характера фазовых переходов. Установлено, что в интервалах $0.0 \leq r \leq 0.2$ и $0.7 \leq r \leq 1.0$ наблюдается фазовый переход первого рода, а в интервале $0.3 \leq r \leq 0.6$ в системе наблюдаются фрустрации.

Ключевые слова: фрустрации, фазовая диаграмма, метод Монте-Карло, модель Поттса.

DOI: 10.21883/FTT.2021.05.50812.270

\section{1. Введение}

В настоящее время возрастает интерес к исследованию фазовых переходов (ФП), критических, магнитных и термодинамических свойств магнетиков, открывающие широкие перспективы для практического применения [1-3]. Модели Поттса и Изинга являются одними из решеточных моделей, используемые для описания физических свойств таких магнетиков. Эти модели также описывают большой класс реальных физических систем: слоистые магнетики, пленки жидкого гелия, сверхпроводящие пленки, адсорбированные пленки и др $[1,4,5]$.

На сегодняшний день, двумерная модель Изинга достаточно хорошо изучена и, практически, все ее свойства известны [4-8]. Иначе обстоит дело с двумерной моделью Поттса. В последние годы исследованию спиновых систем, описываемых моделью Поттса на треугольной решетке было посвящено значительное число работ [4,9-13], в которых были получены ответы на многие вопросы. В работах [9-12] представлены результаты, полученные для двумерной модели Поттса с числом состояний спина $q=2$ и $q=3$ на треугольной решетке и на решетке кагоме. Результаты, представленные в этих работах, показывают, что при определённых значениях величины взаимодействия вторых ближайших соседей данная модель становится фрустрированной. Значение величины взаимодействия вторых ближайших соседей, при котором система становится фрустрированной зависит от числа состояний спина $q$ и от геометрии решетки.

Подобные исследования для двумерной модели Поттса с числом состояний спина $q=4$ на гексагональной решетке до сих пор не проводились. Данная модель интересна тем, что значение $q=4$ является граничным значением интервала $2 \leq q \leq 4$, где наблюдается ФП второго рода и области значений $q>4$, в котором наблюдается фазовый переход первого рода [12].

В работах $[14,15]$ было показано, что в двумерной ферромагнитной модели Поттса с числом состояний спина $q=4$ на гексагональной решетке при учете обменных взаимодействий первых ближайших соседей наблюдается ФП первого рода. Учет антиферромагнитных взаимодействий вторых ближайших соседей приводит к появлению фрустрации и нарушению магнитного упорядочения.

Изменение величины взаимодействия вторых ближайших соседей в этой модели может привести к конкуренции обменных взаимодействий между первыми ферромагнитными и вторыми антиферромагнитными соседями. Конкуренция обменных взаимодействий приводит к фрустрации. Эффекты фрустрации играют важную роль в магнитных системах. Кроме того, фрустрированные спиновые системы проявляют свойства, отличные от соответствующих нефрустрированных систем, что вызывает повышенный интерес к изучению явлений фрустрации в магнитных системах [16-18].

Интерес к модели Поттса с числом состояний спина $q=4$ на гексагональной решетке обусловлен следующими основными причинами.

Во-первых, природа ФП и термодинамические свойства модели Поттса зависят от числа состояний спина $q$ и от геометрии решетки и этот вопрос все еще недостаточно полно изучен. 

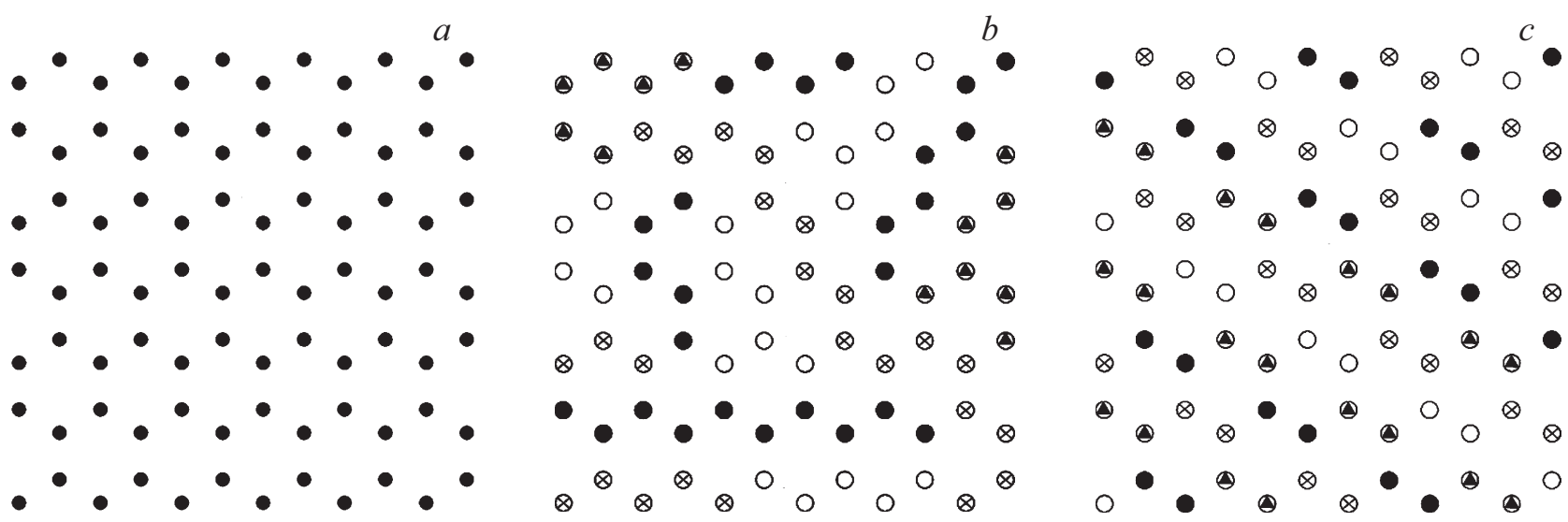

Рис. 1. Магнитные структуры основного состояния.

Во-вторых, исследований ФП и термодинамических свойств модели Поттса с числа состояний спина $q=4$ на гексагональной решетке для различных значений величины взаимодействия вторых ближайших соседей практически нет.

В связи с этим, в настоящей работе нами проводится исследование этой модели в широком интервале значений величины взаимодействия вторых ближайших соседей. Исследование рассматриваемой модели на основе современных методов и идей позволит получить ответ на ряд вопросов, связанных с ФП и термодинамическими свойствами фрустрированных спиновых систем и систем с конкурирующими обменными взаимодействиями.

\section{2. Модель и метод исследования}

Гамильтониан модели Поттса с числом состояний $q=4$ с учетом взаимодействий первых и вторых ближайших соседей может быть представлен в следующем виде $[19,20]$ :

$$
H=-J_{1} \sum_{i, j} \cos \theta_{i, j}-J_{2} \sum_{i, k} \cos \theta_{i, k},
$$

где $J_{1}$ и $J_{2}$ - параметры обменных ферро- $\left(J_{1}>0\right)$ и антиферромагнитного $\left(J_{2}<0\right)$ взаимодействия соответственно для первых и вторых ближайших соседей, $\theta_{i, j}, \theta_{i, k}-$ углы между взаимодействующими спинами $S_{i}-S_{j}$ и $S_{i}-S_{k}, r=\left|J_{2} / J_{1}\right|-$ величина взаимодействия вторых ближайших соседей. В данной работе рассматривается интервал значений $0.0 \leq r \leq 1.0$.

В настоящее время такие системы на основе микроскопических гамильтонианов успешно изучаются на основе метода МК [21-24]. В последнее время разработано много новых вариантов алгоритмов метода МК. Одним из наиболее эффективных для исследования подобных систем является алгоритм Ванга-Ландау метода МК [25], особенно в низкотемпературной области. Поэтому нами, в данном исследовании был использован этот алгоритм.
В стандартный алгоритм Ванга-Ландау нами были внесены дополнения, которые позволяют выяснить магнитную структуру основного состояния системы. Более подробно алгоритм Ванга-Ландау изложен в работах [11]. Данный алгоритм позволяет рассчитать значения термодинамических параметров при любой температуре. В частности, внутреннюю энергию, свободную энергию $F$, теплоемкость $C$ и энтропию $S$ можно вычислить, используя следующие выражения:

$$
\begin{gathered}
U(T)=\frac{\sum_{E} E g(E) e^{-E / k_{B} T}}{\sum_{E} g(E) e^{-E / k_{B} T}} \equiv\langle E\rangle_{T}, \\
F(T)=-k_{B} T \ln \left(\sum_{E} g(E) e^{-E / k_{B} T}\right), \\
C=\left(N K^{2}\right)\left(\left\langle U^{2}\right\rangle-\langle U\rangle^{2}\right), \\
S(T)=\frac{U(T)-F(T)}{T},
\end{gathered}
$$

где $K=\left|J_{1}\right| / k_{B} T, N-$ число частиц, $T-$ температура (здесь и далее температура дана в единицах $\left|J_{1}\right| / k_{B}$ ), $U-$ внутренняя энергия ( $U$ является нормированной величиной).

Для анализа характера ФП нами был использован метод кумулянтов Биндера четвертого порядка и гистограммный метод анализа данных метода МК [26,27].

Расчеты проводились для систем с периодическими граничными условиями и линейными размерами $L \times L=N, L=12 \div 96$.

\section{3. Результаты моделирования}

На рис. 1 представлены магнитные структуры основного состояния для значений $r=0.0,0.3$ и 0.8 . На этом рисунке спины обозначены различными кружками. Спины, обозначенные одинаковыми кружками, имеют одинаковое направление. Как видно на рис. 1, $a$, для 

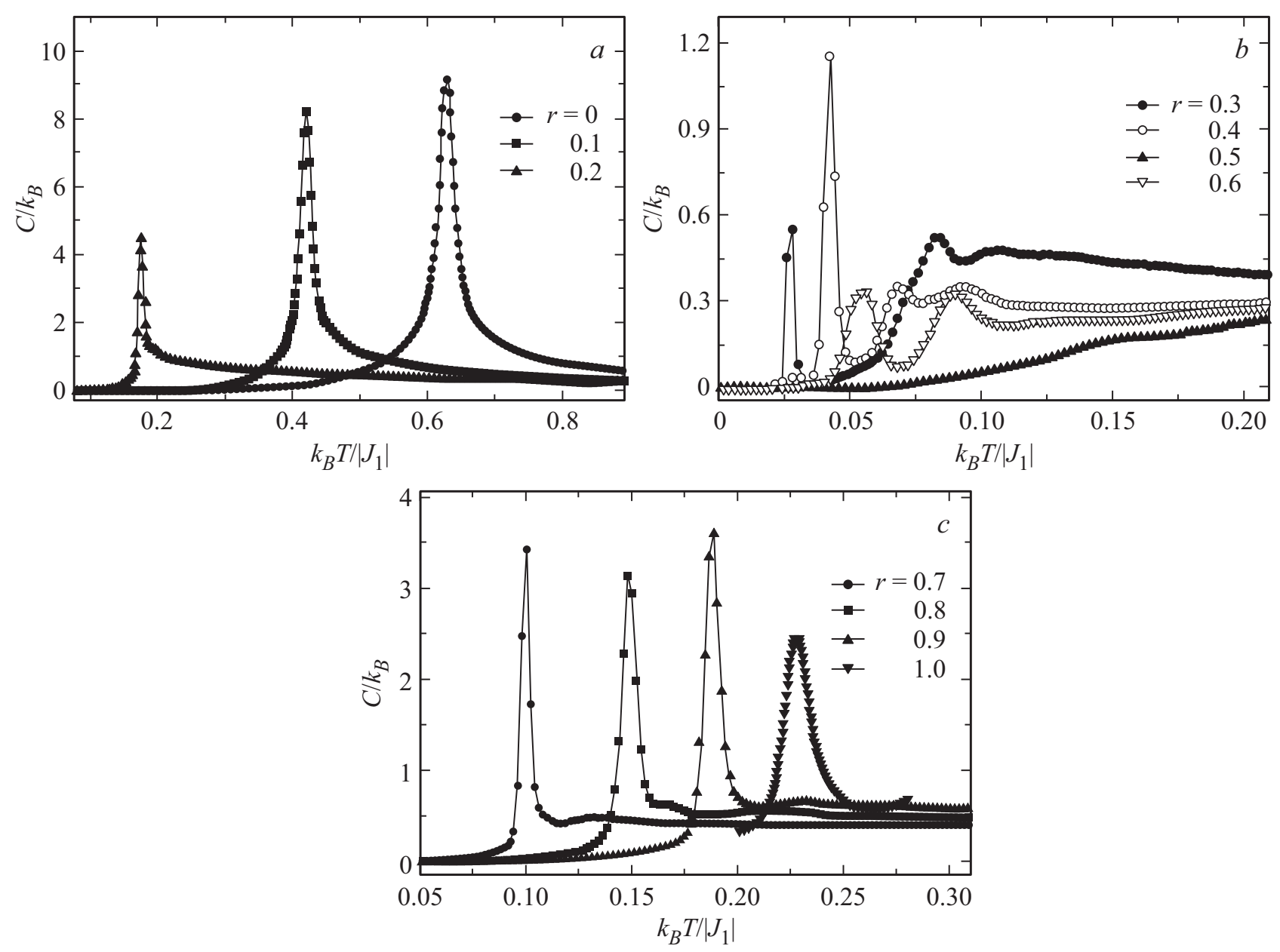

Рис. 2. Температурные зависимости теплоемкости $C / k_{B}$.

значения $r=0.0$ в системе наблюдается ферромагнитное упорядочение. Такая же картина наблюдается для значений $r=0.1$ и $r=0.2$. При $r=0.3$ ферромагнитный порядок нарушается и наблюдаются области с частичным упорядочением (рис. $1, b)$. Аналогичная картина наблюдается в диапазоне $0.3 \leq r \leq 0.6$. В диапазоне $0.7 \leq r \leq 1.0$ наблюдается димерное упорядочение, которое представлено на рис. $1, c$ для $r=0.8$.

На рис. 2 представлены температурные зависимости теплоемкости для разных значений $r$. Как видно из рисунка для всех значений $r$ в диапазонах $0.0 \leq r \leq 0.2$ и $0.7 \leq r \leq 1.0$ вблизи критической точки наблюдаются отчетливые максимумы. В интервале $0.0 \leq r \leq 0.2$ рост $r$ сопровождается со сдвигом максимумов в сторону низких температур и уменьшением значения максимумов. Противоположная картина наблюдается в интервале $0.7 \leq r \leq 1.0$, где максимумы смещаются в сторону высоких температур. Для значений $r=0.3$, $0.4,0.5$ и 0.6 наблюдается расщепление теплоемкости. Расщепление теплоемкости является особым свойством присущее фрустрированным моделям, где вблизи точки фрустрации наблюдается острый пик и куполообразный максимум. Такое поведение теплоемкости позволяет говорить о том, что для значений $r=0.3,0.4,0.5$ и 0.6 в данной модели возникают фрустрации.

Зависимости энтропии $S$ от температуры представлены на рис. 3. Как видно на рисунке в диапазоне $0.0 \leq r \leq 0.2$ в низкотемпературной области энтропия стремится к нулю. Это значит, что в данном диапазоне $r$ отсутствует вырождение основного состояния и система не является фрустрированной. В диапазоне $0.3 \leq r \leq 1.0$ энтропия стремится к отличному от нуля значению. Такое поведение энтропии свидетельствует о том, что в диапазоне $0.3 \leq r \leq 1.0$ наблюдается вырождение основного состояния и возможно возникновение в системе фрустраций.

Фазовая диаграмма зависимости критической температуры от величины взаимодействия вторых ближайших соседей приведена на рис. 4. На диаграмме наблюдаются три различные фазы: ферромагнитная, парамагнитная и димерная. На диаграмме видно, что в интервале значений $0.3 \leq r \leq 0.6$ критическая температура равна нулю и ФП отсутствует. Это объясняется тем, что конкуренция обменных взаимодействий первых и вторых ближайших соседей в данной модели приводит к возникновению полной фрустрации. Фрустрации нарушают порядок в системе и приводит к исчезновению ФП. 

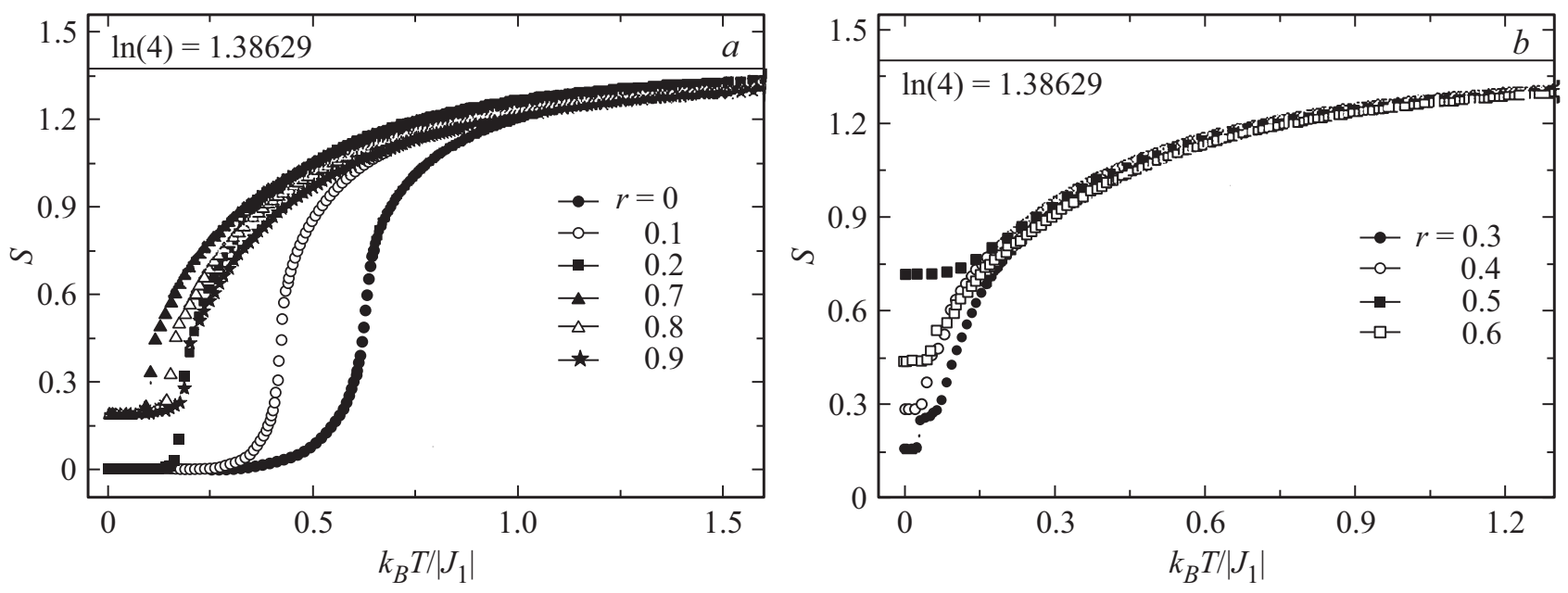

Рис. 3. Температурные зависимости энтропии $S$.

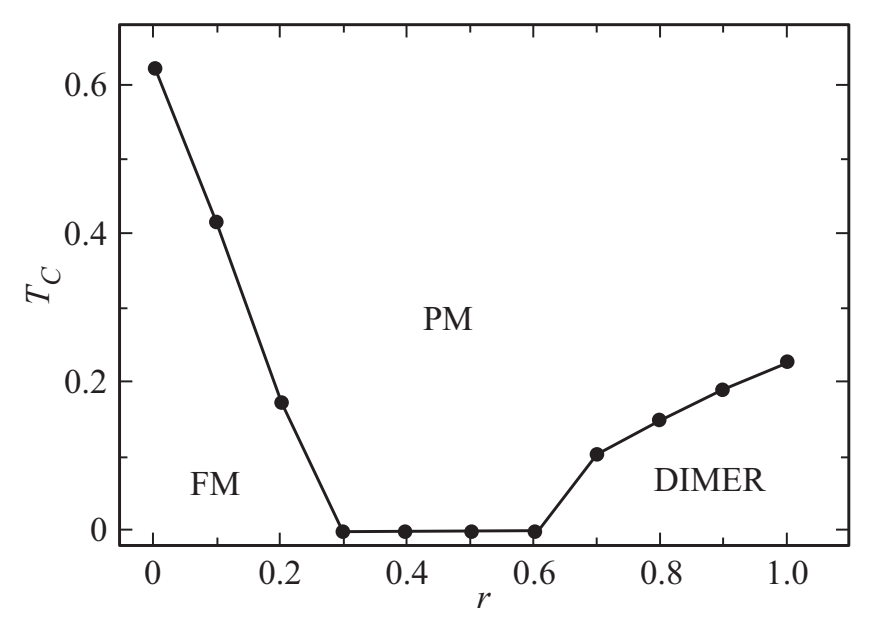

Рис. 4. Фазовая диаграмма зависимости критической температуры от величины взаимодействия вторых ближайших соседей.

Для анализа характера ФП и определения критической температуры ТС в диапазонах $0.0 \leq r \leq 0.2$ и $0.7 \leq r \leq 1.0$ нами использовался метод кумулянтов Биндера четвертого порядка [27]:

$$
\begin{gathered}
V_{L}=1-\frac{\left\langle U^{4}\right\rangle_{L}}{3\left\langle U^{2}\right\rangle_{L}^{2}}, \\
U_{L}=1-\frac{\left\langle m^{4}\right\rangle_{L}}{3\left\langle m^{2}\right\rangle_{L}^{2}},
\end{gathered}
$$

где $V_{L}-$ энергетический кумулянт, $U_{L}-$ магнитный кумулянт.

Выражения (6) и (7) позволяют определить критическую температуру $T_{C}$ с большой точностью для ФП первого и второго рода соответственно. Следует отметить, что применение кумулянтов Биндера позволяет также хорошо тестировать тип ФП в системе. Известно, что ФП первого рода характеризуются тем, что величина $V_{L}$ стремится к некоторому нетривиальному значению $V^{*}$ согласно выражению

$$
V_{L}=V^{*}+b L^{-d}
$$

при $L \rightarrow \infty$ и $T=T_{C}(L)$, где величина $V^{*}$ отлична от $2 / 3$, а минимальная величина $U_{L \min }\left(T=T_{\min }\right)$ расходится $U_{L \min }\left(T=T_{\min }\right) \rightarrow \infty$ при $L \rightarrow \infty$.

В случае ФП второго рода кривые температурной зависимости кумулянтов Биндера $U_{L}$ имеют четко выраженную точку пересечения [27].

Как видно из рис. 5 температурные зависимости $U_{L}$ для $r=0.1$ при различных $L$ в критической области не пересекаются в одной точке. Это свидетельствует в пользу наличия в данной модели ФП первого рода. Температурная зависимость энергетического кумулянта $V_{L}$ для разных значений $L$ представлена на рис. 6 . Как видно из графика величина $V_{L}$ стремится к 2/3, а величина $V^{*}=2 / 3$, что характерно для ФП второго

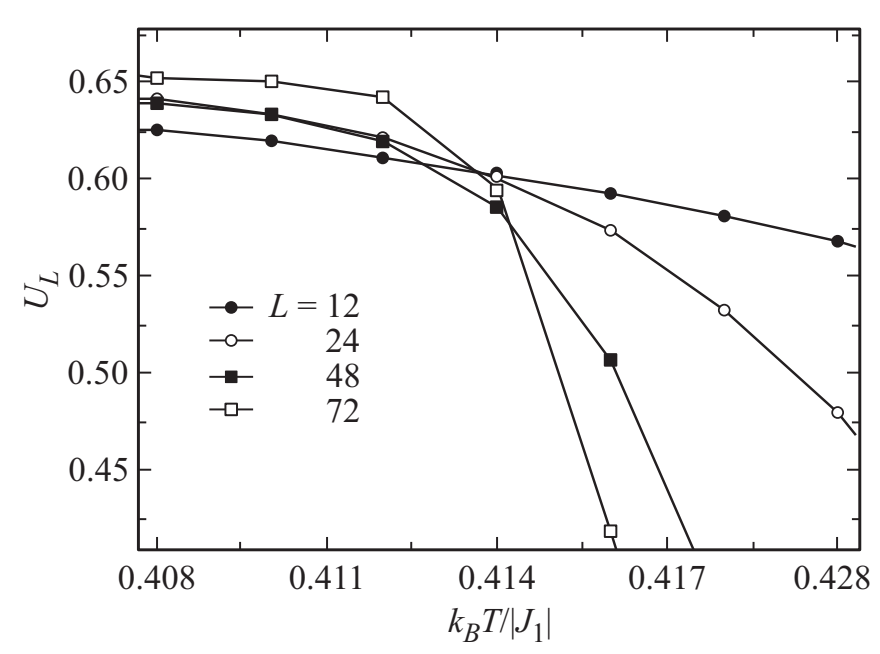

Рис. 5. Температурные зависимости магнитного кумулянта Биндера $U_{L}$. 


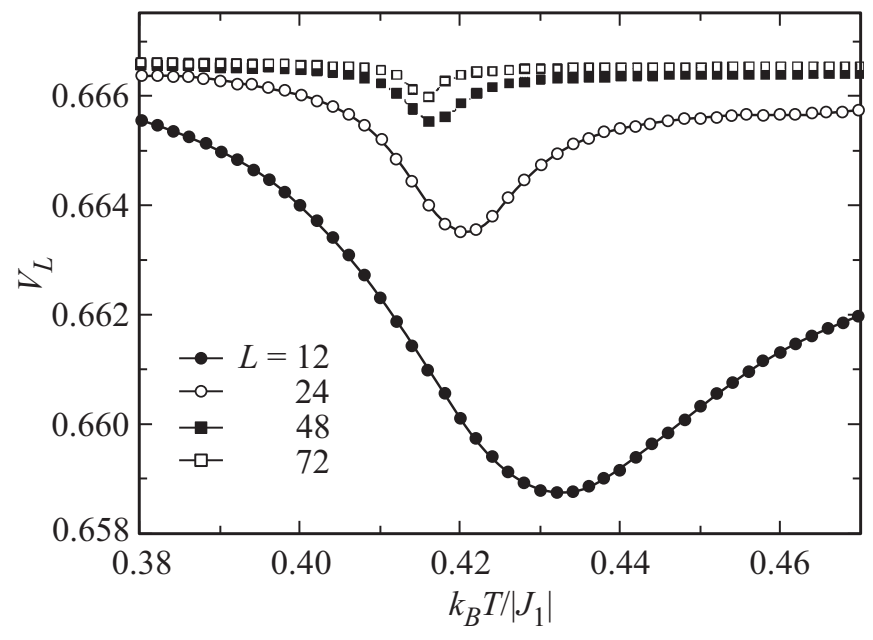

Рис. 6. Температурные зависимости энергетического кумулянта Биндера $V_{L}$.

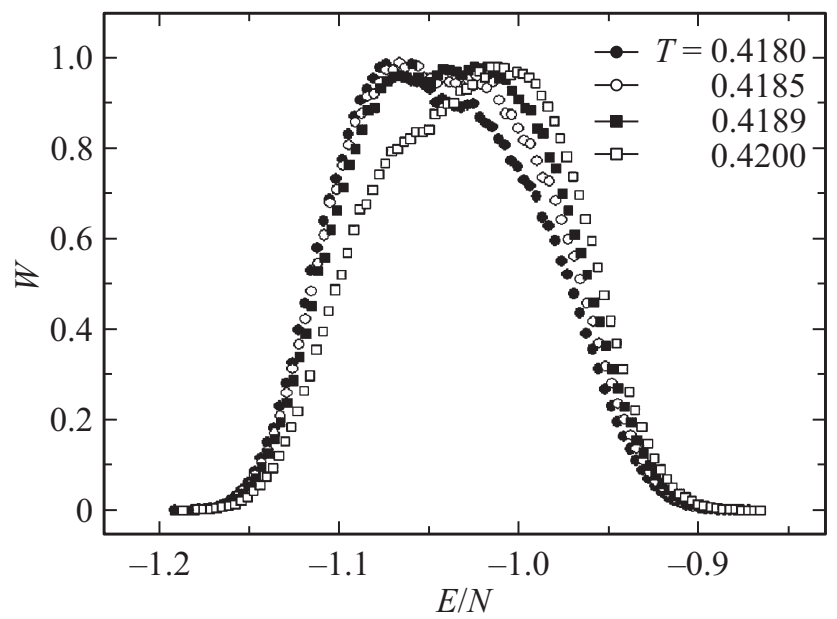

Рис. 7. Гистограммы распределения энергии для $L=96$ при различных температурах.

рода. Эта величина рассчитана используя выражение (8). Аналогичная картина наблюдается для значений $r$ в диапазонах $0.0 \leq r \leq 0.2$ и $0.7 \leq r \leq 1.0$.

Поэтому, для более подробного анализа рода ФП нами использовался гистограммный анализ данных метода МК $[18,19]$. Этот метод позволяет надежно определить род ФП. Методика определения рода ФП этим методом подробно описана в работах $[26,28]$.

Полученные на основе гистограммного анализа данных результаты показывают, что в данной модели наблюдается ФП первого рода. Это продемонстрировано на рис. 7. На этом рисунке представлены гистограммы распределения энергии для системы с линейными размерами $L=96$ для $r=0.1$. Графики построены при различных температурах близких критической температуре. Из рисунка видно, что в зависимости вероятности $W$ от энергии $E$ для всех температур наблюдаются два максимума, которые свидетельствует о ФП первого рода. Наличие двойного пика на гистограммах распределения энергии является достаточным условием для ФП первого рода. Отметим, что двойные пики на гистограммах распределения для исследуемой модели наблюдаются для значений $r$ в интервалах $0 \leq r \leq 0.2$ и $0.7 \leq r \leq 1.0$. Это позволяет нам утверждать о том, что в рассмотренных интервалах значений $r$ наблюдаются ФП первого рода.

Результаты настоящей работы, полученные для значения $r=0.0$ согласуются с данными полученными нами ранее для двумерной модели Поттса с числом состояний спина $q=4$ на треугольной решетке [18] и на решетке кагоме [29]. Исследование ФП данной модели в широком интервале значений $r$ в литературе не встречается.

\section{4. Заключение}

Исследование магнитных структур основного состояния, фазовых переходов и термодинамических свойств двумерной модели Поттса с числом состояний спина $q=4$ на гексагональной решетке с учетом взаимодействий первых и вторых ближайших соседей выполнено с использованием алгоритма Ванга-Ландау метода Монте-Карло. Получены магнитные структуры основного состояния в широком интервале значений величины взаимодействия вторых ближайших соседей. Построена фазовая диаграмма зависимости критической температуры от величины взаимодействия вторых ближайших соседей. Показано, что в интервалах $0.0 \leq r \leq 0.2$ и $0.7 \leq r \leq 1.0$ наблюдается фазовый переход первого рода. В интервале $0.3 \leq r \leq 0.6$ наблюдается вырождение основного состояния, и система становится фрустрированной.

\section{Финансирование работы}

Исследование выполнено при финансовой поддержке Российского фонда фундаментальных исследований в рамках научных проектов 19-02-00153-а.

\section{Конфликт интересов}

Авторы заявляют, что у них нет конфликта интересов.

\section{Список литературы}

[1] H.T. Diep. Frustrated Spin Systems. World Scientific Publishing Co. Pte. Ltd., Singapore (2004). P. 624.

[2] R.J. Baxter. Exactly Solved Models in Statistical Mechanics. Academic, N. Y. (1982), Mir, Moscow (1985).

[3] F.Y. Wu. Exactly Solved Models: A ourney in Statistical Mechanics. World Scientific, New Jersey (2008).

[4] F.Y. Wu. Rev. Mod. Phys. 54, 235 (1982).

[5] W. Zhang, Y. Deng. Phys. Rev. E 78, 031103 (2008).

[6] А.К. Муртазаев, М.К. Рамазанов, Ф.А. Кассан-Оглы, М.К. Бадиев. ЖЭТФ 144, 1239 (2013).

[7] A.K. Murtazaev, M.K. Ramazanov, M.K. Badiev. Phys. B: Condens. Matter 476, 1 (2015). 
[8] F.A. Kassan-Ogly, A.K. Murtazaev, A.K. Zhuravlev, M.K. Ramazanov, A.I. Proshkin. J. Magn. Magn. Mater. 384, 247 (2015).

[9] M. Nauenberg, D.J. Scalapino. Phys. Rev. Lett. 44, 837 (1980).

[10] J.L. Cardy, M. Nauenberg, D.J. Scalapino. Phys. Rev. B 22, 2560 (1980).

[11] M.K. Ramazanov, A.K. Murtazaev, M.A. Magomedov. Phys. A 521, 543 (2019).

[12] H. Feldmann, A.J. Guttmann, I. Jensen, R. Shrock, S.-H. Tsai. J. Phys. A 31, 2287 (1998).

[13] F.A. Kassan-Ogly, A.I. Proshkin. Phys. Solid State. 60, 1090 (2018).

[14] А.К. Муртазаев, М.К. Рамазанов, М.К. Мазагаева, М.А. Магомедов. ЖЭТФ 156, 502 (2019).

[15] М.К. Рамазанов, А.К. Муртазаев, М.А. Магомедов, М.К. Мазагаева. ФТТ 62, 442 (2020).

[16] М.К. Рамазанов, А.К. Муртазаев. Письма в ЖЭТФ 109, 610 (2019).

[17] А.К. Муртазаев, М.К. Рамазанов, М.К. Бадиев. ФТТ 61, 1898 (2019).

[18] А.К. Муртазаев, Д.Р. Курбанова, М.К. Рамазанов. ФТТ 61, 2195 (2019).

[19] M.G. Townsend, G. Longworth, E. Roudaut. Phys. Rev. B 33 (1986) 4919.

[20] Y. Chiaki, O. Yutaka. J. Phys. A 34, 8781 (2001).

[21] R. Masrour, A. Jabar, A. Benyoussef, M. Hamedoun. J. Mag. Mag. Mater. 410, 223 (2016).

[22] R. Masrour, A. Jabar. Physica A 491, 926 (2018).

[23] R. Masrour, A. Jabar. Physica A 515, 270 (2019).

[24] A.K. Murtazaev, M.K. Ramazanov, M.K. Badiev. Physica A 507, 210 (2018).

[25] F. Wang, D.P. Landau. Phys. Rev. E 64, 0561011-1 (2001).

[26] F. Wang, D.P. Landau. Phys. Rev. Lett. 86, 2050 (2001).

[27] K. Binder, D. Heermann. Monte Carlo Simulation in Statistical Physics: An Introduction. Springer, Berlin, Heidelberg (2010).

[28] М.К. Рамазанов, А.К. Муртазаев. Письма в ЖЭТФ 106, 72 (2017).

[29] А.К. Муртазаев, Т.Р. Ризванова, М.К. Рамазанов, М.А. Магомедов. ФТТ 62, 1278 (2020).

Редактор Т.Н. Василевская 